\title{
Presentación \\ Economía global y desarrollo en tiempos de pandemia: los retos para América Latina y el Caribe
}

\author{
Alicia Bárcena y Mario Cimoli \\ Editores invitados
}

\section{Una edición especial para tiempos complejos}

La edición especial de la Revista CEPAL que presentamos ha sido elaborada en el contexto de la crisis sanitaria ocasionada en 2020 por la propagación de la enfermedad por coronavirus (COVID-19) a escala mundial. Junto con esta pandemia se ha desencadenado también la más aguda crisis económica del capitalismo global desde la Gran Depresión de la década de 1930. En términos más amplios, se trata de una grave crisis humanitaria que ha costado millones de vidas humanas, truncadas por el contagio del virus, así como pérdidas de producción y empleo que se han traducido también en deterioro de las condiciones de bienestar material de vastos segmentos de la población mundial (sobre todo en materia de salud, educación y nutrición) y, por lo mismo, en fuertes retrocesos en materia de pobreza y distribución de los ingresos. En esta sombría escena mundial, América Latina ha resultado ser una de las regiones más afectadas por la pandemia, situación que no es casual en el marco de las asimetrías internas y externas que configuran su disfuncional estilo de desarrollo, patrón que las literaturas estructuralista y neoestructuralista han descrito en numerosos escritos en los que se analiza la dinámica socioeconómica y ambiental de la región en diferentes estadios históricos ${ }^{1}$.

\section{Editores invitados}

Alicia Bárcena es Secretaria Ejecutiva de la Comisión Económica para América Latina y el Caribe (CEPAL). Correo electrónico: alicia.barcena@un.org.

Mario Cimoli es Secretario Ejecutivo Adjunto de la Comisión Económica para América Latina y el Caribe (CEPAL). Correo electrónico: mario.cimoli@un.org.

\footnotetext{
1 Por mencionar solo algunos, véanse Prebisch (1962), Bielschowsky (1998), Ffrench-Davis y Torres (2021) y CEPAL (2020).
} 
En el contexto de la historia sanitaria mundial, la pandemia es un fenómeno aún en curso cuyos efectos son difíciles de determinar y dimensionar cualitativa y cuantitativamente. Antes de esta crisis sanitaria, otras enfermedades de carácter pandémico habían generado altos niveles de mortalidad año tras año en las últimas décadas (Žižek, 2020). Cabe preguntarse, entonces, qué diferencia esta pandemia de otros flagelos similares. Quizás una respuesta plausible se relacione con la velocidad de contagio y la mortalidad que ha provocado este virus en su primer año de circulación. Dado lo anterior, la pandemia ha tenido un efecto inmediato sobre la fuerza de trabajo y, por ende, sobre la demanda efectiva, la producción y el comercio. Además, ha evidenciado que la desigualdad dentro de los países en desarrollo constituye un obstáculo significativo para desplegar políticas de contención sanitaria.

Diversos analistas han orientado sus esfuerzos a estudiar el impacto de este fenómeno sanitario mundial y esbozar distintas perspectivas sobre el devenir de la economía en el contexto de la pospandemia. Un ejemplo de ello es la reflexión de Chena y Pérez Caldentey (2020), quienes plantean la pregunta referida a si la pandemia acentuará las tendencias imperantes en la forma de organización y articulación de la producción a escala mundial y las finanzas globales o si, por el contrario, será un factor disruptivo y de destrucción creativa. Más allá de las distintas respuestas que puedan surgir frente a esta interrogante, lo cierto es que esta crisis sanitaria tendrá efectos inevitables en la estructura productiva de la región y en las posibilidades de llevar adelante un proceso de cambio estructural y distributivo. Mitigar sus impactos negativos en el corto plazo con medidas urgentes que también consideren el largo plazo es el camino para que estos efectos conduzcan a superar los problemas estructurales que enfrenta la región, en particular su rezago tecnológico y productivo, y la elevada desigualdad.

\section{El contenido de esta edición}

Con estas premisas, la presente edición especial de la Revista CEPAL ofrece distintas visiones respecto de los efectos del COVID-19 en la región y plantea, de acuerdo con los enfoques de los autores convocados, variadas propuestas de políticas orientadas a superar la crisis sanitaria, social y económica que ha ocasionado la propagación del virus.

El material que presentamos puede clasificarse según las distintas perspectivas de análisis planteadas en los 14 artículos que incluye la edición. En tal sentido, resaltan artículos en que se aborda la problemática del COVID-19 desde una perspectiva global. Este es el caso de los trabajos de Ha-Joon Chang, Giovanni Dosi, Maria Savona, y Nora Lustig y Mariano Tommasi. Esta edición especial incluye otro conjunto de artículos en que se exploran los efectos de la pandemia desde una perspectiva regional, compuesto por los ensayos de Alicia Bárcena y Mario Cimoli; José Antonio Ocampo; Martín Abeles, Esteban Pérez Caldentey y Gabriel Porcile; Nicole Bidegain, Lucía Scuro e lliana Vaca Trigo, y Juan Carlos Moreno Brid y Rodrigo Morales. Se agregan a este grupo de ensayos un par de artículos en que se analizan tópicos de la pandemia combinando las perspectivas global, regional y nacional. En esta categoría se ubican los ensayos de Benedicte Bull y Francisco Robles y de José Gabriel Palma, que además destacan los desafíos de economía política que conlleva la pandemia. Se completa el conjunto de artículos de esta edición con tres trabajos en que se analizan casos nacionales, el de Leonardo Lomelí sobre México, el de Luiz Carlos Bresser-Pereira sobre el Brasil y el de Ramón López y Gino Sturla sobre Chile.

En relación con los artículos de perspectiva global, el trabajo de Chang aborda un tema central y recurrente dentro de los debates del desarrollo: el multilateralismo. El autor analiza los factores que han conducido al deterioro del sistema mundial de comercio en el contexto del orden económico neoliberal que ha hegemonizado la gobernanza de la economía global desde la década de 1980. Plantea que dicho deterioro podría estar dando paso a un "nuevo" nuevo orden económico internacional, derivado no solo del desplome del comercio mundial y los efectos aún vigentes de la crisis económica de 2008, 
sino también de cambios en la economía mundial y en las ideas dominantes, además de otros factores como la irrupción de China como potencia económica mundial, el cambio climático y la crisis sanitaria generada por la propagación del COVID-19.

En el artículo de Dosi, en tanto, se analizan algunas tendencias del capitalismo mundial anteriores a la pandemia y ciertos aspectos de ella que plantearían una encrucijada entre mantener el modo de gobernanza vigente en los países (caracterizado por lo que autor denomina tecnoautoritarismo) o avanzar hacia nuevas formas de organización social. El autor sostiene además que la crisis sanitaria se ha producido en un contexto de creciente tecnologización y fragilidad socioeconómica. En dicho contexto, surgen tendencias muy claras relacionadas con el deterioro de la distribución funcional del ingreso en desmedro del salario, la ampliación de brechas entre la productividad y los salarios y el crecimiento explosivo de las tasas de beneficios (financieros y no financieros).

En una clave similar, Savona examina los efectos del COVID-19 en el contexto de las transformaciones digitales y las estructuras laborales presentes actualmente en la economía mundial, planteando algunos de los nuevos desafíos de políticas que surgen a partir de la pandemia. En su análisis, la autora argumenta que la "nueva normalidad" que surgirá debe reconocer una "nueva esencialidad" en relación con el mundo del trabajo y los sectores de actividad económica. El conjunto de ensayos de perspectiva global se completa con el trabajo de Lustig y Tommasi, quienes analizan el vínculo entre los efectos del COVID-19 y la protección social requerida por los segmentos más vulnerables de la población.

Respecto de los artículos de perspectiva regional, este número ofrece al lector una diversidad de enfoques, datos e implicaciones de políticas que posibilitan ampliar la comprensión de este fenómeno sanitario en nuestra región. En el ensayo de Bárcena y Cimoli, a partir de la premisa inicial de que América Latina y el Caribe ha sido una de las regiones más afectadas por la pandemia, se analizan las asimetrías estructurales anteriores a la crisis sanitaria, que venían evidenciando la disfuncionalidad del proceso de crecimiento de los países de la región, y se plantea la necesidad de avanzar hacia un nuevo estilo de desarrollo.

En una línea similar de análisis, Abeles, Pérez Caldentey y Porcile examinan los efectos del COVID-19 en la región, argumentando que para lograr la recuperación se requerirán políticas fiscales de gran magnitud dirigidas a estimular la demanda efectiva. La composición de estos esfuerzos fiscales deberá, según los autores, ponderar fuertemente el componente de inversión orientado a la generación de capacidades tecnológicas, la diversificación productiva y mayores encadenamientos de la matriz productiva.

El ensayo de Ocampo ofrece un análisis de los impactos del COVID-19 en clave histórica, mediante la comparación de la actual crisis sanitaria con la Gran Depresión de la década de 1930, la crisis asiática de 1997 y la crisis del período 2008-2009, que el autor denomina crisis del Atlántico Norte. El autor señala que todos los choques asociados a financiamiento externo, términos de intercambio, volúmenes de comercio y niveles de remesas han tenido impactos menos graves que la actual contingencia sanitaria. En el centro de estos resultados, Ocampo releva la insuficiente cooperación financiera internacional. Desde esta óptica, afirma que la fuerza con que ha golpeado la pandemia en América Latina y el Caribe obedece más bien a los factores estructurales internos que redundaron en el estancamiento económico que se observaba en la región antes de la crisis, en concordancia con los análisis realizados en los artículos de Bárcena y Cimoli y de Abeles, Pérez Caldentey y Porcile.

El conjunto de ensayos de perspectiva regional se completa con el trabajo de Bidegain, Scuro y Vaca Trigo, quienes analizan el impacto de la pandemia desde el enfoque de género, enfatizando en la forma como ha afectado la autonomía económica de las mujeres en la región, y el artículo de Moreno Brid y Morales, quienes ofrecen una perspectiva subregional de los efectos del COVID-19 en el caso de Centroamérica. 
En cuanto a los artículos que combinan distintas visiones, mencionamos en primer lugar el ensayo de Bull y Robles, quienes exploran, mediante una cuidadosa revisión de literatura, el modo en que las pandemias inciden sobre la desigualdad en la distribución de los ingresos. Los autores sostienen que la literatura económica proporciona evidencia significativa en el sentido de que estos fenómenos acrecientan la desigualdad distributiva, en tanto que los estudios historiográficos y de la politología plantean que las pandemias pueden generar quiebres institucionales que, en un contexto de mutaciones dentro de las élites y de presiones de demandas populares, pueden tener como resultado el mantenimiento de los niveles de inequidad. Tras contrastar estas tesis teóricas con un conjunto de datos empíricos presentados para el caso de la región, los autores sugieren que en el contexto de la pandemia de COVID-19, si bien se observan transferencias de ingresos hacia los sectores más ricos y ciertos cambios en la composición de las élites, de todos modos, al ser estas las que han llevado adelante la conducción política durante la crisis sanitaria, existen pocos indicios de que la región esté asistiendo a un quiebre institucional.

En una línea similar de análisis, Palma aborda la problemática de la desigualdad en la región, con énfasis en el caso de Chile. Recurriendo al enfoque de la economía política de David Ricardo, comprende la distribución del ingreso como la articulación de un conflicto caracterizado por múltiples actores sociales en pugna por diversos intereses, disputas que se dan en un contexto histórico, político e institucional determinado que resulta tan relevante como los "fundamentos" puramente económicos. A partir de este marco analítico, Palma sostiene que, sin importar los hechos ocurridos a lo largo de la historia regional, los ricos de América Latina muestran una fuerte persistencia en esa condición, en virtud de la ley de hierro de las oligarquías, es decir, mediante un mecanismo que permite la reconstrucción de instituciones disfuncionales, que aseguran un patrón distributivo que favorece la posición de los sectores oligárquicos.

Finalmente, el número especial se completa con tres artículos en que se exploran los efectos de la pandemia de COVID-19 a partir de casos nacionales. Lomelí ofrece un análisis de la economía de la salud en México, Bresser-Pereira examina el impacto que ha tenido la pandemia en el caso de la economía brasileña y López y Sturla presentan un ensayo referido a la tributación sobre los grandes patrimonios, enfocando su análisis en el caso de Chile.

\section{Reflexiones que surgen en torno a estos ensayos}

Los trabajos presentados en esta edición especial plantean aspectos que van más allá de la emergencia sanitaria ocasionada por el COVID-19. Si bien los ensayos compilados se refieren a los efectos generados por la pandemia, según lo reseñado en la sección anterior, también abordan aspectos estructurales anteriores a la crisis tanto a nivel regional como mundial. En este sentido, la crisis sanitaria releva algunas interrogantes recurrentes sobre la economía mundial cuando se ha visto enfrentada a choques globales de magnitud similar.

Durante las últimas cuatro décadas, a medida que el proceso de globalización se fue intensificando en términos tecnológicos, financieros y comerciales, la economía mundial fue afectada de manera cada vez más recurrente por crisis económicas que han agudizado las brechas entre países desarrollados y en desarrollo, la desigualdad dentro de los países y entre ellos, las tensiones políticas y las amenazas reales del deterioro ambiental.

Estos hechos, particularmente acentuados por la crisis económica de 2008, han puesto en evidencia los múltiples fallos de la globalización en términos de su gobernanza y de los patrones disfuncionales de desarrollo que ha generado en los países. Rodrik (2011) ha abordado estos aspectos, analizando las tensiones entre el proceso de globalización, el avance democrático y las políticas nacionales de 
desarrollo. En este contexto, el papel que desempeñan las instituciones resulta crucial para alcanzar un equilibrio adecuado entre las tres variables en juego, que es por naturaleza complejo, dadas la diversidad y la heterogeneidad de arreglos a los que llegan los diferentes países de acuerdo con las especificidades y preferencias sociales. Muchas veces estos arreglos nacionales difieren de las formas institucionales que requieren los mercados en términos de regulación, estabilidad y legitimación. La desregulación de los mercados financieros condujo a la economía global a ciclos más marcados de auge y recesión y, finalmente, a nuevas crisis financieras, que han agudizado las condiciones socioeconómicas dentro de los países, promoviendo a la vez movimientos sociales contrarios a la globalización.

En el plano de las relaciones centro-periferia, la supremacía de los mercados financieros desregulados por sobre los sectores reales de la economía mundial ha tenido profundos efectos en la capacidad de los países, sobre todo de los países en desarrollo, para llevar adelante políticas productivas y de inserción que les permitan cerrar sus brechas internas y externas. En este sentido, los problemas de la gobernanza financiera mundial encuentran un símil en las formas de organización del sistema multilateral de comercio, con restricciones que subordinan las capacidades del Sur global de implementar políticas industriales nacionales de orden desarrollista (Chang, 2002).

Frente a estas crisis económicas recurrentes como consecuencia de una globalización disfuncional en términos de gobernanza y efectos, un año después de que Rodrik publicara su obra Paradoja de la globalización, Paul Krugman daba a conocer un manifiesto cuya proclama central era la de "idetener esta recesión ahora!". En este trabajo, plantea que el sesgo recesivo presente en el capitalismo mundial desde 2008 , hoy devenido en crisis no solo económica, sino también humanitaria en su más amplio sentido, como consecuencia del COVID-19, radica en la persistencia de las políticas de austeridad adoptadas por las tecnocracias, que inhiben los estímulos fiscales y monetarios que, a juicio de Krugman y otros economistas keynesianos, son necesarios para impulsar los motores de demanda agregada que se requieren para reactivar la demanda agregada mundial (Krugman, 2012). En diversos estudios de la Comisión Económica para América Latina y el Caribe (CEPAL) publicados durante los últimos diez años se ha abordado el sesgo recesivo anterior a la pandemia, planteando como uno de sus factores fundamentales la insuficiencia de la demanda efectiva en términos globales y regionales. CEPAL (2016) es un ejemplo de ello, con su idea de promover una eficiencia keynesiana dentro del contexto del sistema multilateral.

Durante estos años de agudización de las contradicciones reveladas por el sistema económico mundial vigente, la CEPAL refinó sus propuestas de avance económico, social y ambiental mediante una estrategia de desarrollo basada en un enfoque de derechos. La hora de la igualdad: brechas por cerrar, caminos por abrir (CEPAL, 2010) fue el documento que planteó los fundamentos de esta estrategia, que en muchos casos antecedió a los planteamientos críticos del modelo de globalización vigente en el mundo elaborados por los más destacados economistas del campo heterodoxo a nivel mundial.

A partir de los planteamientos expresados en ese documento, se fueron ampliando y detallando los contenidos de esta estrategia de desarrollo y búsqueda de igualdad en materias que han constituido de manera permanente los mensajes desarrollistas de la Comisión: el cambio estructural para el desarrollo inclusivo (CEPAL, 2012) y la necesidad de promover pactos amplios para el desarrollo, como los referidos a la fiscalidad, la política productiva, el avance de las mujeres, la cohesión social y el desarrollo ambientalmente sostenible, entre otros (CEPAL, 2014). Destaca también la vinculación de esta estrategia con la Agenda 2030 para el Desarrollo Sostenible y los Objetivos de Desarrollo Sostenible (CEPAL, 2016), así como los documentos La ineficiencia de la desigualdad (CEPAL, 2018), en el que se argumenta de manera sólida sobre la invalidez de "la gran disyuntiva" (the big trade-off) entre igualdad y eficiencia planteada por Okun (1975), y Construir un nuevo futuro: una recuperación transformadora con igualdad y sostenibilidad (CEPAL, 2020), en el que se plantea la propuesta más reciente de la Comisión, contextualizada en los efectos de la pandemia, que considera las brechas que enfrenta la región en materia económica, social y ambiental y se expresa en un conjunto de 
lineamientos de políticas que posibilitarían una recuperación acompañada de un cambio estructural y de inclusión social.

Así, mientras la CEPAL brindó estas visiones, que abordan las asimetrías estructurales clave que retardan el desarrollo en la región, entre ellas las brechas tecnológicas y productivas que limitan su crecimiento y la cultura del privilegio (una interpretación original que resume el puzle de economía política que explica la imposibilidad de reducir la desigualdad en un sentido amplio y aplicar una política tributaria progresiva), otras voces en el mundo se han referido a los efectos nocivos del patrón de globalización vigente. En este sentido surgió la amplia producción de Thomas Piketty y su interpretación del capitalismo en el siglo XXI, que brinda datos de concentración de la riqueza de larga data en el caso de las economías desarrolladas y puso en el debate y en la investigación económica el análisis de la distribución del ingreso en los percentiles más elevados, particularmente en el 1\% más alto (Piketty, 2014). Por su parte, Milanovic (2016) instaló en el debate sobre la distribución el concepto de desigualdad global, es decir, el diferencial de ingresos medios entre los habitantes del planeta. Según este economista, la desigualdad en términos globales obedece a los ciclos de Kuznets de la dinámica distributiva dentro de los países y de la convergencia de ingresos entre ellos. Si bien se trata de un aporte a las investigaciones en estas materias, las tesis de Milanovic generan cierta controversia entre los analistas, algunos de los cuales señalan que la construcción institucional y el reparto asimétrico del poder dentro de las sociedades a favor de las élites es un aspecto que cumple un papel más preponderante que los que plantea Milanovic. Los trabajos de Palma y de Bull y Robles incluidos en este número ofrecen una visión orientada en esta línea argumental.

Además de los nuevos debates sobre la distribución que se presentan en esta edición especial, los trabajos incluidos abren también una reflexión en torno a otros temas centrales. Uno de ellos es el papel que debe recuperar el Estado orientado a políticas de desarrollo, toda vez que los mercados dejados a su libre arbitrio -en el espíritu de la filosofía liberal del laissez faire- no han logrado por sí solos, especialmente en el mundo en desarrollo, operar con eficacia para satisfacer los requerimientos de un crecimiento vigoroso con una adecuada distribución de sus beneficios entre los distintos actores sociales. En CEPAL (2020) se aborda el papel que le cabe al Estado en cuanto agente generador de pactos para la implementación de políticas macroeconómicas, industriales, sociales y ambientales que viabilicen el cambio estructural sostenible y la inclusión social.

En concordancia con estas ideas, varios economistas han planteado la necesidad de ampliar la función del Estado en las políticas económicas y de desarrollo. De manera muy destacada, Mariana Mazzucato ha reivindicado el papel emprendedor del sector público en estas políticas. Ha señalado concretamente que el Estado ha sido un actor clave en materia de inversiones y que su rol en esta función ha quedado invisibilizado por el sector privado de alta tecnología, segmento sobre el cual se han generado mitos que opacan el papel del sector público (Mazzucato, 2014). A partir de este planteamiento, vinculó el papel del Estado con la importancia de la innovación tecnológica, retomando un aspecto central de la economía política clásica: la teoría del valor. La interrogante central de Mazzucato (2019) al respecto es ¿qué es la riqueza y de dónde procede el valor? Más aún, se pregunta de forma acertada qué es realmente el valor y cuánto de lo que se presenta como tal no es acaso apropiación o extracción de valor preexistente. Estas preguntas por cierto son muy relevantes para las perspectivas de desarrollo en nuestra región, pues los procesos extractivistas que caracterizan las matrices productivas de sus economías en muchos casos concentran valor y no necesariamente crean riqueza o la acrecientan.

Por consiguiente, la necesidad de avanzar en la creación de nuevos sectores o industrias que generen valor genuino otorga un papel preponderante al Estado en dicho objetivo, entendiéndose que la política industrial y el cambio estructural constituyen misiones dirigidas a satisfacer necesidades estratégicas para las sociedades (Mazzucato, 2021), como la seguridad alimentaria y sanitaria, y otros sectores esenciales. Estos planteamientos son ciertamente convergentes con lo que ha propuesto la CEPAL (2020) en relación con los sectores productivos de alto potencial para desplegar una nueva 
estrategia de desarrollo (energías renovables, movilidad sostenible y espacios urbanos, manufacturas para el cuidado de la salud y bioeconomía, entre otros). Los trabajos de Dosi y Savona incluidos en esta edición aportan elementos para la reflexión de estas cuestiones.

Retornando a la pregunta de cuál será el devenir de la economía mundial después de la pandemia, hay diversas reflexiones en torno a ella, muchas de las cuales antecedieron en muy poco tiempo la irrupción de la pandemia, que ponen de manifiesto los malestares que venía desencadenando la dinámica del capitalismo global. Se ha aludido a varios de ellos en esta sección. El año 2019 fue muy prolífico en cuanto a producción escrita sobre esto. En primer lugar, cabe mencionar la publicación Capital e ideología, de Thomas Piketty, quien plantea que la verdadera esencia de la desigualdad no es puramente económica o tecnológica, sino política e ideológica. En una clave más cercana a la de Gramsci que a la de Marx, sostiene que el motor de la historia no es la lucha de clases, sino las disputas hegemónicas en torno a la ideología. Una reflexión importante de Piketty es que el capitalismo puede ser superado para dar paso a sociedades más justas en sistemas políticos de tipo socialista-participativo o social-federativo (Piketty, 2019).

Por otro lado, en tónicas orientadas más bien a corregir las asimetrías del patrón capitalista vigente -es decir, superar el neoliberalismo- se sitúan las visiones de Milanovic (2019) y Stiglitz (2019). Milanovic plantea que el capitalismo, único sistema imperante en la economía mundial desde la década de 1990, presenta notables fallos, como la desigualdad y la corrupción, pero también ventajas en términos de satisfacción de la autonomía de los individuos, aunque advierte que ha tenido el costo moral del "éxito material" como el objetivo final de estos (el individualismo). A pesar de ello, es optimista en cuanto al devenir de este sistema y argumenta que existen posibilidades de reducir sus excesos e inequidades, algo similar a lo que Stiglitz (2019) conceptualiza como un "capitalismo progresista" que puede surgir después de corregir aspectos como la concentración corporativa global, los fallos de la industria financiera, la captura de los Estados nacionales por parte de los grupos económicos y la concentración de datos que surge al amparo de los nuevos conglomerados tecnológico-digitales. La acción sobre estas áreas, a juicio de Stiglitz, puede ayudar a reconstruir los cimientos de un capitalismo más justo e inclusivo.

Un punto de vista más matizado, quizás más pesimista que los planteamientos de Piketty, Stiglitz y Milanovic, es el que expresó Rodrik en 2020, en los momentos más difíciles de la pandemia. Su crítica al manejo de la crisis sanitaria se logra imbricar con los problemas previos que arrastraba la economía mundial, sistematizados en su tríada globalización-democracia-Estado, en el marco de la hegemonía neoliberal. Señala Rodrik:

En resumen, el COVID-19 tal vez no altere-y mucho menos revierta- las tendencias evidentes antes de la crisis. El neoliberalismo seguirá su muerte lenta. Los autócratas populistas se volverán aún más autoritarios. La hiperglobalización continuará a la defensiva mientras los estados-nación reclaman espacio para implementar políticas. China y Estados Unidos se mantendrán en su curso de colisión. Y la batalla dentro de los estados-nación entre oligarcas, populistas autoritarios e internacionalistas liberales se intensificará, mientras la izquierda lucha por diseñar un programa que apele a una mayoría de votantes (Rodrik, 2020).

En estas sentencias, Rodrik evidentemente reafirma el declive de la fase neoliberal del capitalismo, pero apunta de forma realista a que los sesgos recesivos que generó durante la última década y que la pandemia agudizó continuarán operando en los años venideros con una persistencia significativa. No obstante lo señalado por Rodrik, lo que resulta evidente es la necesidad creciente de persistir en la idea de poner todos los esfuerzos intelectuales, políticos y civiles al servicio de una globalización más equilibrada, justa e inclusiva y, junto con ello, volcar esos mismos esfuerzos en una región, la nuestra, que avance en la superación de sus obstáculos estructurales al desarrollo y la igualdad. 
En este sentido, los artículos que se presentan en esta edición especial de la Revista CEPAL ofrecen al lector un cúmulo de visiones y reflexiones que esperamos le permitan ampliar su comprensión sobre los fenómenos globales y regionales que habrá que enfrentar a partir de este nuevo choque mundial que ha significado la irrupción del COVID-19.

\section{Bibliografía}

Bielschowsky, R. (1998), "Evolución de las ideas de la CEPAL", Revista de la CEPAL, № extraordinario (LC/G.2037-P), Santiago, Comisión Económica para América Latina y el Caribe (CEPAL), octubre.

CEPAL (Comisión Económica para América Latina y el Caribe) (2020), Construir un nuevo futuro: una recuperación transformadora con igualdad y sostenibilidad (LC/SES.38/3-P/Rev.1), Santiago, octubre. (2018), La ineficiencia de la desigualdad (LC/SES.37/3-P), Santiago, mayo.

(2016), Horizontes 2030: la igualdad en el centro del desarrollo sostenible (LC/G.2660/Rev.1), Santiago. (2014), Pactos para la igualdad: hacia un futuro sostenible (LC/G.2586(SES.35/3)), Santiago.

(2012), Cambio estructural para la igualdad: un enfoque integrado del desarrollo (LC/G.2524 (SES.34/3)), Santiago.

(2010), La hora de la igualdad: brechas por cerrar, caminos por abrir (LC/G.2432(SES.33/3)), Santiago.

Chang, H-J. (2002), Kicking away the ladder, development strategy in historical perspective, Anthem Pr.

Chena, P. y E. Pérez Caldentey (2020), "Structural change and counter-reforms in Latin America: necessity or possibility?", Cuadernos de Economía, vol. 39, № 80, Universidad Nacional de Colombia.

Ffrench-Davis, R. y M. Torres (2021), "Neo-structuralism", The New Palgrave Dictionary of Economics, M. Vernengo, E. Pérez Caldentey y J. Barkley Rosser (eds.), Palgrave Macmillan.

Krugman, P. (2012), End this Depression Now!, W. W. Norton \& Company.

Mazzucato, M. (2021), Mission Economy: A Moonshot Guide to Changing Capitalism, Harper Business. (2019), El valor de las cosas. ¿Quién produce y quién gana en la economía global?, Taurus. (2014), El Estado emprendedor: mitos del sector público frente al privado, RBA.

Milanovic, B. (2019), Capitalism, Alone: the Future of the System that Rules the World, The Belknap Press of Harvard University Press.

- (2016), Global Inequality: A New Approach for the Age of Globalization, The Belknap Press of Harvard University Press.

Okun, A. (1975), Equality and Efficiency: The Big Tradeoff, Brooking Institutions Press.

Piketty, T. (2019), Capital e ideología, Paidós. (2014), El capital en el siglo XXI, Ciudad de México, Fondo de Cultura Económica.

Prebisch, R. (1962), "El desarrollo económico de la América Latina y algunos de sus principales problemas", Boletín económico de América Latina, vol. 7, № 1, febrero.

Rodrik, D. (2020), “¿El COVID-19 reconstruirá al mundo?” [en línea] https://www.project-syndicate.org/ commentary/will-covid19-remake-the-world-by-dani-rodrik-2020-04/spanish?barrier=accesspaylog. (2011), The Globalization Paradox: Democracy and the Future of the World Economy, W. W. Norton \& Company.

Stiglitz, J. (2019), Capitalismo progresista: la respuesta a la era del malestar, Taurus.

Žižek, S. (2020), Pandemia: la COVID-19 estremece al mundo, Editorial Anagrama. 